\title{
Joint Multicast Routing and Channel Assignment in Multiradio Multichannel Wireless Mesh Networks using Tabu Search
}

\author{
Hui Cheng \\ Department of Computer Science \\ University of Leicester \\ Leicester LE1 7RH, United Kingdom \\ Email: hc118@le.ac.uk
}

\author{
Shengxiang Yang \\ Department of Computer Science \\ University of Leicester \\ Leicester LE1 7RH, United Kingdom \\ Email: sy11@le.ac.uk
}

\begin{abstract}
This paper proposes a tabu search (TS) based optimization approach to search a minimum-interference multicast tree which satisfies the end-to-end delay constraint and optimizes the usage of the scarce radio network resource in wireless mesh networks. The path-oriented encoding method is adopted and each candidate solution is represented by a tree data structure (i.e., a set of paths). Since we expect the multicast trees on which the minimum-interference channel assignment can be produced, a fitness function that returns the total channel conflict is devised. The techniques for controlling the tabu search procedure are well developed. A simple yet effective channel assignment algorithm is proposed to reduce the channel conflict. Simulation results show that the proposed TS multicast algorithm can produce the multicast trees which have better performance in terms of both the total channel conflict and the tree cost than that of a well known multicast algorithm in wireless mesh networks.
\end{abstract}

Index Terms-wireless mesh network; multicast; channel assignment; tabu search;

\section{INTRODUCTION}

Wireless mesh networks (WMNs) [1] have emerged as a new paradigm of static multi-hop wireless networks. A typical wireless mesh network consists of two types of wireless nodes, i.e., mesh routers and mobile clients. Each mesh router functions as both a relay node and an access point. As a relay node, a mesh router can forward packets to other mesh routers according to the routing information. As an access point, a mesh router can forward packets from or to the mobile clients which are currently associated with it. Mesh routers are stationary with power supply while clients may roam and change the associated mesh routers. In the wireless mesh networks, all the mesh routers are self-organized to establish ad hoc networks and maintain the network topology. As a result, WMNs have the advantages of easy deployment, high reliability, and large coverage. There is an increasing interest in using WMNs to provide ubiquitous network connectivity in enterprises, campuses, and in metropolitan areas [2].

Multicast [3] is an important network service, which is the delivery of information from a source to multiple destinations simultaneously using the most efficient strategy to deliver the messages over each link of the network only once, creating copies only when the links to the destinations split. It provides underlying network support for collaborative multimedia applications such as multimedia conference, distant education and content distribution. Quality of Service (QoS) requirements [3] proposed by different multimedia applications are often versatile. Among them, end-to-end delay [4] is a pretty important QoS metric since real-time delivery of multimedia data is often required. The multicast tree cost, used to evaluate the utilization of network resource, is also an important QoS metric especially in wireless networks where limited radios and channels are available. However, little work has addressed QoS multicast in WMNs.

In WMNs, if two mesh routers falling into the radio transmission range want to enable the communication link between them, they must tune their radios to the same channel. However, the wireless interference occurs when two links whose distance is less than 2 hops away are assigned to the same channel to support the concurrent communications, which is termed as channel conflict [5]. The heavy interference caused by channel conflict degrades the performance of the wireless communication severely. Therefore, for multicast routing, each link on the multicast tree requires to be assigned to one channel and the assignment should lead to minimum interference. Therefore, the QoS multicast routing in WMNs involves not only to search a routing tree but also to assign proper channels to its links. In fact, the minimum-interference channel assignment problem itself is basically the Max $K$-cut problem [2], which is known to be NP-hard. Since our problem is the routing tree construction plus minimum-interference channel assignment, it is also NP-hard.

So far the QoS multicast routing has not drawn much attention from the research community of WMNs. However, it is believed that efficient multicast, which cannot be readily achieved through combined unicast or simplified broadcast, is essential to WMNs and deserves a thorough investigation [6]. In this paper, we propose an efficient QoS multicast routing algorithm in WMNs, which utilizes a well-designed tabu search (TS) [7] algorithm to search a low cost routing tree on which the channel assignment can produce the minimum 
interference. The idea is that for each searched delay-bounded multicast tree, we first assign channels to its links by the proposed channel assignment algorithm, and then evaluate it by the total channel conflict and tree cost. Since the channel assignment strategy is fixed, intuitively by examining more candidate routing trees, we can find the one on which the minimum interference channel assignment can be achieved. Hence, the strong search capability of TS can be well utilized to solve this problem.

\section{RELATED WORK}

Similar as mobile ad hoc networks (MANETs) [8], a wireless mesh network is also a type of self-organizing wireless network. However, there are three main differences between them. First, nodes in MANETs are often moving while mesh routers in WMNs are normally stationary. Second, in MANETs all the mobile nodes work in a peer-to-peer fashion and each node forwards packets on behalf of other nodes, while in WMNs a hierarchy is formed where mesh routers form a backbone and mesh clients can only access their associated mesh routers. Third, a mobile node in MANETs is normally equipped with one radio while a mesh router in WMNs is equipped with at least two radios.

In MANETs, a number of multicast routing protocols, using a variety of basic routing algorithms and techniques, have been proposed over the past few years [8]. However, they mainly focus on the discovery of the optimal multicast forwarding structure (i.e., tree or mesh) spanning mobile nodes and do not need to consider the channel assignment problem. In MANETs, since a mobile node may be equipped with a Global Positioning System (GPS) device, geographical information can also be utilized for route discovery. Therefore, according to the type of the utilized information, the multicast routing protocols in MANETs can be classified as topological routing and geographical routing.

In WMNs, little work has been done on multicast routing due to its intractability. In [6], two multicast algorithms were proposed, which first build a multicast tree between the source and receivers, and then use dedicated strategies to assign channels to the tree aiming to reduce interference. However, since both algorithms separate the construction of the multicast tree and the channel assignment, they bear a potential drawback, that is, channel assignment cannot work well with the determined multicast tree. Furthermore, they do not consider the delay constraint which is a common issue for multicast problems.

Tabu search is a meta-heuristic that can lead a local search procedure to explore the solution space beyond local optimality. Tabu search uses a local or neighbourhood search procedure to iteratively move from a solution $x$ to a solution $x$ ' in the neighbourhood of $x$, until some stopping criterion has been satisfied. Compared with other meta-heuristics such as genetic algorithm and simulate annealing, tabu search is more general and conceptually much simpler. However, TS still shows comepeting performance when it is used for solving many combinatorial optimization problems. Tabu search has been applied to the QoS multicast routing in the wired networks such as the multimedia communication networks [3], [9].

In [3], the binary encoding is adopted where each bit of the binary string corresponds to a different node in the network. For each binary string, a graph $\mathrm{G}^{\prime}$ is derived from the network topology $G$ by including all the nodes appearing in the string and the links connecting these nodes. Then the minimum spanning tree $T$ of $\mathrm{G}^{\prime}$ acts as the candidate multicast tree represented by the binary string. This encoding method is a bit complicated and each binary string cannot directly represent the candidate solution. A multicast tree is a union of the routing paths from the source to each receiver. Hence, it is a more natural choice to adopt the path-oriented encoding method [10], [11] than the binary encoding.

In [9], the path-oriented encoding is adopted. For each destination, a sink tree is constructed by connecting it to the source and all the other destinations using the shortest (i.e., least-cost) paths. On the sink tree, each path from the tree root to a leaf node is named as a superpath. Each iteration the tabu search algorithm first generates a few neighbors of a multicast tree by replacing its one superpath using a few randomly selected superpaths separately. Then, among these new neighbors, the one with the best cost is selected, and considered as the new solution for the next iteration. If a superpath is deleted at one iteration, then reintroducing the same superpath to the current tree is tabu. Assuming $m$ is the number of the destinations, there are $m$ sink trees. Each candidate solution is just one combination of $m$ paths from the $m$ sink trees. Therefore, the size of the candidate solution space is limited by all the sink trees. The performance of the algorithm is hindered by the limited solution space to be explored.

We are not aware of any other work that jointly considers multicast routing, which further consists of channel assignment as well as QoS in multiradio multichannel wireless mesh networks, although there are quite a few works that are related to some relevant aspects. Since TS shows good performance in the wired networks, we believe its strong search capability can also help find a low cost low interference routing tree in wireless mesh networks. However, to our best knowledge, TS has not been addressed to solve the multicast problems in WMNs.

\section{PROBLEM Formulation}

In this section, we first present our network model and then formulate the problem of joint QoS multicast routing and channel assignment.

We consider a wireless mesh network with stationary mesh routers where each router is equipped with a certain number of radio network interface cards (NICs). We model a wireless mesh network by a undirected and connected topology graph $G(V, E)$, where $V$ represents the set of mesh routers and $E$ represents the set of communication links connecting two neighboring mesh routers falling into the radio transmission range. A communication link $(i, j)$ can not be used for 
packet transmission until both node $i$ and node $j$ have a radio interface each with a common channel. In addition, message transmission on a wireless communication link will experience a remarkable delay.

For clarity of presentation, we assume the binary interference model, i.e., two communication links either interfere or do not interfere. Given the binary interference model, the set of pairs of communication links that interfere with each other over the same channel can be represented by a conflict graph [5]. A communication link in the topology graph corresponds to a vertex in the conflict graph. With the binary interference model, the conflict graph $G_{c}\left(V_{c}, E_{c}\right)$ can be easily derived from the topology graph $G(V, E)$. We assume the communication links $(a, b)$ and $(c, d)$ in the topology graph $G(V, E)$ are represented by the node $i_{c}$ and node $j_{c}$ in the conflict graph $G_{c}\left(V_{c}, E_{c}\right)$, respectively. Then if the minimum distance between $(a, b)$ and $(c, d)$ is less than 2 hops, we have $\left(i_{c}, j_{c}\right) \in E_{c}$.

Here, we summarize some notations that we use throughout this paper.

- $G(V, E)$, the WMN topology graph.

- $G_{c}\left(V_{c}, E_{c}\right)$, the conflict graph derived from the WMN topology graph.

- $K=\{0,1,2, \ldots, k\}$, the set of available orthogonal channels

- $s$, the source node of the multicast communication.

- $R=\left\{r_{0}, r_{1}, \ldots r_{m}\right\}$, the set of receivers of the multicast communication.

- $T\left(V_{T}, E_{T}\right)$, a multicast tree with nodes $V_{T}$ and links $E_{T}$.

- $V_{T}^{\text {Leaf }}$, the set of leaf nodes on the tree $T$.

- $P_{T}\left(s, r_{i}\right)$, a path from $s$ to $r_{i}$ on the tree $T$.

- $d_{l}$, the delay on the communication link $l$.

- $I_{T}(f)$, the total channel conflict on the tree $T$ under the channel assignment $f$.

- $C_{T}$, the cost of the tree $T$.

The problem of joint QoS multicast routing and channel assignment in a multiradio multichannel wireless mesh network can be informally described as follows. Given a network of mesh routers with multiple radio interfaces, a delay upper bound, a source node and a set of receivers, we wish to find a delay-bounded multicast tree and assign a unique channel to each communication link on the tree. We define the total channel conflict as the number of pairs of communication links on the tree that are interfering (i.e., are assigned the same channel and are connected by an edge in the conflict graph). The objective of our problem is to minimize the above defined total channel conflict, as it results in improving the system throughput [6].

We also want to optimize the usage of the scarce network resources in the multicast tree. So we define the tree cost as the number of the radio interfaces involved in the multicast communications. We aim to find a multicast tree with low cost. There are two factors related to the tree cost. One is the number of communication links on the tree. Each communication link has one sender and one receiver, thereby occupying two radio interfaces. So we should reduce the number of links on the multicast tree, which also helps reduce the multicast end-to-end delay. The other factor is the number of broadcast nodes generated from the channel assignment. We make all the branch nodes become broadcast nodes by exploiting wireless multicast advantage (WMA) [12] and the detail is described in Section 4.2. If there are several multicast trees which have the same channel conflict value, we will choose the one with the minimum tree cost.

More formally, consider a wireless mesh network $G(V, E)$ and a multicast communication request from the source node $s$ to a set of receivers $R$ with the delay upper bound $\Delta$. The joint $Q o S$ multicast routing and channel assignment problem is to find a multicast tree $T\left(V_{T}, E_{T}\right)$ satisfying the delay constraint as shown in (1) and compute a function $f: E_{T} \rightarrow K$ defined in (2) to minimize the total channel conflict $I_{T}(f)$ defined in (3).

$$
\begin{aligned}
& \max _{r_{i} \in R}\left\{\sum_{l \in P_{T}\left(s, r_{i}\right)} d_{l}\right\} \leq \Delta . \\
& f\left(i_{c} \in E_{T}\right)=\{j \mid j \in K\}
\end{aligned}
$$$$
I_{T}(f)=\left|\left\{\left(i_{c}, j_{c}\right) \in E_{c} \mid f\left(i_{c}\right)=f\left(j_{c}\right), i_{c} \in E_{T}, j_{c} \in E_{T}\right\}\right| .
$$

Since the source only transmits packets and all the leaf nodes only receive packets, each of them occupies one radio interface only. All the other nodes are branch nodes which need to do both the transmission and reception. So each branch node occupies two radio interfaces. As a result, the tree cost $C_{T}$ is calculated as follows:

$$
C_{T}=|\{s\}|+\left|V_{T}^{\text {Leaf }}\right|+2 *\left(\left|V_{T}\right|-|\{s\}|-\left|V_{T}^{\text {Leaf }}\right|\right) .
$$

\section{Algorithm Design}

This section describes the proposed TS based joint QoS multicast routing and channel assignment algorithm. The TS operations consist of several key components: solution representation, initial solution, neighborhood structure, fitness function, tabu move, tabu list, aspiration criterion, and termination rule. Solutions are expressed by tree data structure. Note that every step guarantees that a multicast tree does not violate the delay constraint. The channel assignment algorithm aims to achieve the minimum interference on a given multicast tree.

We adapt TS to the joint multicast routing and channel assignment problem, and the objective function is just the fitness function, which returns the total channel conflict of the multicast tree. First, the initial solution is generated randomly to explore the diversity. For the current solution, one of its neighbors is determined by the random path replacement. Then TS moves from the current solution to its neighbor, even this move deteriorates the fitness value. To explore more unvisisted solutions, solutions that have been recently visited are tabu for a few iterations. An aspiration criterion is proposed to free the solutions in tabu status to continue the search. When the number of continuous iterations without improving the current optimal solution reaches the specified upper bound, 


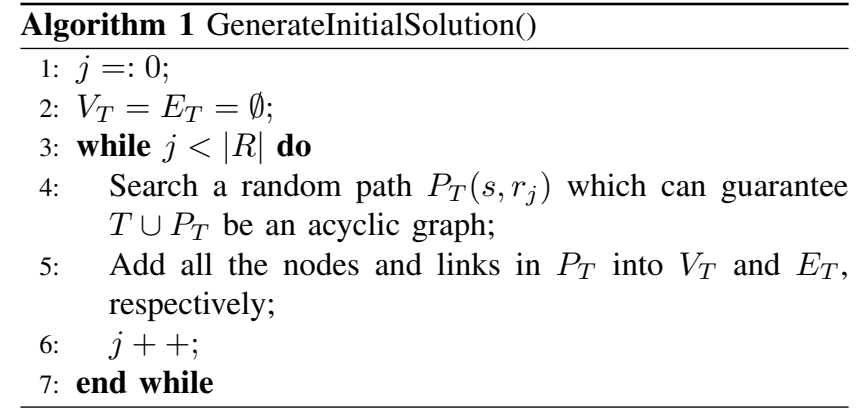

the algorithm ends and outputs the best solution that TS has ever visited as the final solution.

\section{A. Design of Tabu Search}

1) Solution Representation: A routing path is encoded by a string of positive integers that represent the IDs of nodes through which the path passes. Each locus of the string represents an order of a node. The first locus is for the source and the last one is for the receiver. The length of a routing path should not exceed the maximum length $|V|$, where $V$ is the set of nodes in the WMN.

For a multicast tree $T$ spanning the source $s$ and the set of receivers $R$, there are $|R|$ routing paths all originating from $s$. Therefore, we encode a tree by an integer array in which each row encodes a routing path along the tree. For example, for $T$ spanning $s$ and $R$, row $i$ in the corresponding array $A$ lists up node IDs on the routing path from $s$ to $r_{i}$ along $T$. Therefore, $A$ is an array of $|R|$ rows. All the solutions are encoded under the delay constraint. In case it is violated, the encoding process is usually repeated so as to satisfy the delay constraint.

2) Initial Solution: To explore the solution diversity, in the initial solution $Q$, all the routing paths are randomly generated. We start to search a random path from $s$ to $r_{i} \in R$ by randomly selecting a node $v_{1}$ from $N(s)$, the neighborhood of $s$. Then we randomly select a node $v_{2}$ from $N\left(v_{1}\right)$. This process is repeated until $r_{i}$ is reached. Thus, we get a random path $P_{T}(s$, $\left.r_{i}\right)=\left\{s, v_{1}, v_{2}, \ldots, r_{i}\right\}$. Since no loop is allowed on the multicast tree, the nodes that are already included in the current tree are excluded, thereby avoiding reentry of the same node. The initial solution is generated as follows.

3) Fitness Function: Given a solution, we should accurately evaluate its quality (i.e., fitness value), which is determined by the fitness function. In our algorithm, we aim to find a low cost multicast tree on which the minimum interference channel assignment can also be achieved. Our primary criterion regarding solution quality is the total channel conflict and the subsidiary one is the tree cost. Therefore, among a set of candidate solutions (i.e., multicast trees) with the same minimum channel conflict value, we choose the one with the lowest tree cost. The fitness value of chromosome $C h_{i}$ (representing multicast tree $T)$, denoted as $F\left(C h_{i}\right)$, is given by:

$$
F\left(C h_{i}\right)=\left[I_{T}(f)+1.0\right]^{-1} .
$$

The proposed fitness function only involves the total channel conflict. As mentioned above, The tree cost is used in the course of selecting the elitism for recording the searched optimal solution.

4) Neighborhood Structure: Since TS performs searching from one solution to one of its neighbors in the neighborhood, we need to determine the neighborhood structure of each solution. In accordance with the solution representation, we propose the following method to construct the neighborhood. First, randomly select one receiver $r_{i}$ from $R$, and randomly select another node $v_{i}$ on the path $\left(s \longrightarrow r_{i}\right)$. Then replace the subpath $\left(v_{i} \longrightarrow r_{i}\right)$ by a new random subpath to generate a neighbor solution. However, the replacement should guarantee that the delay constraint is not violated.

5) Tabu Move: According to the solution representation and the neighborhood structure, each tabu move is a replacement of a subpath from a non-leaf node to a receiver. A new solution is reached after a move. Three cases may appear after each move.

(a) The fitness value of the new solution is greater than that of the original solution. That is, the new solution is superior to the original one.

(b) The fitness value of the new solution is equal to that of the original solution. That is, the new solution has the same quality as the original one in terms of the total channel conflict. However, they may still have different tree costs.

(c) The fitness value of the new solution is less than that of the original solution. That is, the new solution is inferior to the original one.

In the algorithm, each iteration we randomly select one node pair $\left\{v_{1}, r_{1}\right\}$. Then we replace the subpath $\left(v_{1} \longrightarrow r_{1}\right)$ by another different random subpath. Thus, a new solution is generated as a neighbor and its fitness value is calculated.

6) Tabu List: A tabu list is maintained to prevent returning to previously visited solutions. Each iteration we generate one neighbor. Without loss of generality, we assume that the neighbor is generated by replacing $\left(v_{1} \longrightarrow r_{1}\right)$. Then we push the subpath $\left(v_{1} \longrightarrow r_{1}\right)$ into the tabu list. As a result, one subpath is tabu each time. Since the new neighbor is selected, it is necessary to forbid the addition of the subpath $\left(v_{1} \longrightarrow\right.$ $r_{1}$ ), otherwise the solution may return to the previously visited one in the following iterations.

The size of the tabu list is set to $\lfloor|R| / 2\rfloor$, where $R$ is the set of receivers.

7) Aspiration Criterion: Aspiration criterion is a device used to override the tabu status of moves whenever appropriate [9]. It temporarily overrides the tabu status if the move is sufficiently good. In our algorithm, at each iteration a new subpath is generated randomly. However, if the new path is currently in the tabu list, it cannot be used. Then we generate another new subpath randomly. If this new subpath is also in the tabu list, of these two tabu subpaths we will free the one which lies closer to the tabu list head.

8) Termination Rule: In the algorithm, we record the current optimal solution and we also record the number of continuous iterations without improving it. Therefore, the 
termination rule employed is to control the maximum number of continuous iterations without improving the present optimal solution. We calculate the ratio of this number to the total iteration number. If the ratio exceeds the specified upper bound ratio, we believe that to run the algorithm further will not contribute any improvement to the optimal solution. Therefore, we terminate the search to reduce the overhead. In the algorithm, we set ratio to 0.3 .

The maximum number of iterations is given to guarantee that the algorithm will terminate after sufficient search has been done. We denote $W$ as the total number of iterations. As suggested in [9], we set $W$ to 500. We denote $U$ as the upper bound of the continuous iterations without improving the current optimal solution. We have

$$
U=\lambda * W(0<\lambda<1) .
$$

So when $U$ is reached, the algorithm will terminate. In the algorithm, $\lambda \leq 0.3$.

\section{B. Channel Assignment Algorithm}

In a wireless mesh network, a link cannot be used for data transmission until it has been assigned a wireless communication channel. To support the multicast communication over the routing tree, an appropriate channel should be assigned to each link on the tree so as to achieve the minimum interference (i.e., channel conflict). In addition, the number of available channels is limited in the current network protocols. For example, in IEEE 802.11-based wireless networks, there are 11 available channels. However, at most 3 of them are orthogonal (noninterfering). The number of radio interfaces is also limited as a type of scarce radio network resource. Hence the channel assignment should use as small number of channels and radio interfaces as possible.

Since the minimum-interference channel assignment problem is NP-hard, we propose a heuristic algorithm which aims to reduce both the channel conflict and resource utilization. Given the set of orthogonal channels $K=\{0,1, \ldots, k\}(k \geq 2)$, the algorithm works on the multicast tree $T$ as follows.

For each routing path, the algorithm uses 3 channels to do the assignment. Since the minimum distance between two links to avoid channel conflict is 2 hops, 3 is the least number of channels to achieve conflict free assignment on each routing path of the multicast tree. By our assignment strategy, all the links originating from the same branch node are assigned the same channel as utilizes the so-called WMA [12]. WMA refers to that a single transmission can be received by all the nodes that are within the transmission range of a transmitting node. Hence, using one radio interface only, the branch node transmits packets to all its children. This also saves the number of used radio interfaces.

\section{Performance Evaluation}

In this section, the proposed TS based joint multicast routing and channel assignment algorithm is compared with Zeng' Level Channel Assignment (LCA) multicast algorithm [6] through simulation experiments. The LCA multicast algorithm
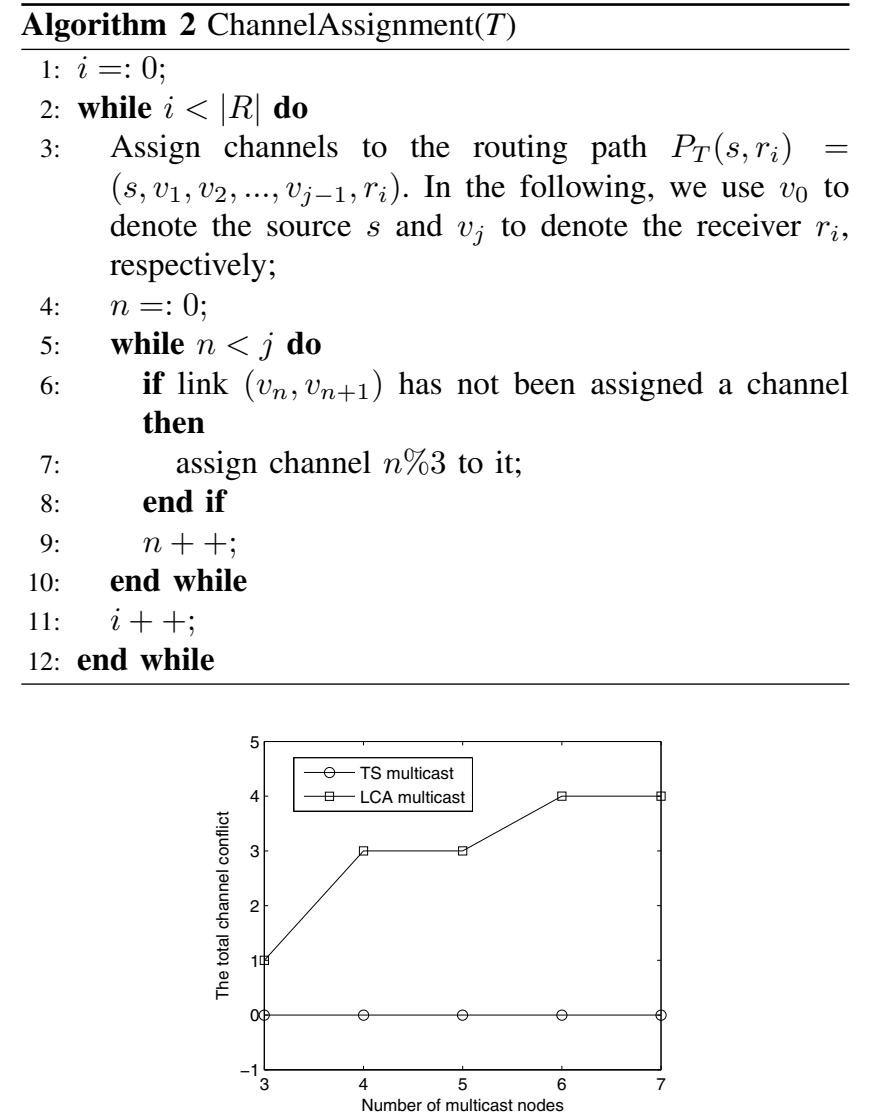

(a)

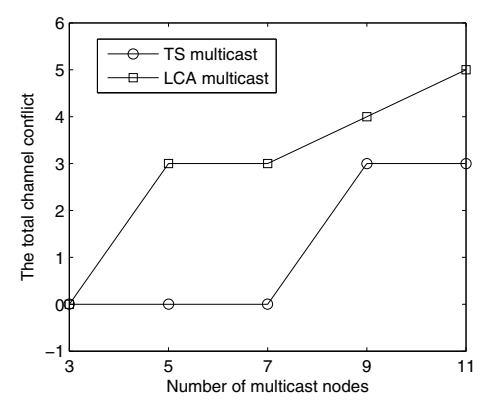

(b)

Fig. 1. Comparison of TS multicast and LCA multicast in terms of the total channel conflict in: (a) a WMN of 11 nodes; (b) a WMN of 23 nodes.

is composed of two components. First, it constructs a multicast tree based on breadth first search (BFS) aiming to minimize the hop count distances between the source and receivers. Second, it uses a dedicated strategy to assign channels to the tree aiming to reduce the interference. Hence, this algorithm separates the multicast tree construction and channel assignment. If the channel assignment strategy cannot work well on the generated multicast tree, the algorithm can do nothing while our algorithm can search other trees.

In our algorithm, the delay upper bound $\Delta$ is set to 20 . Each experiment is terminated when the upper bound of the 


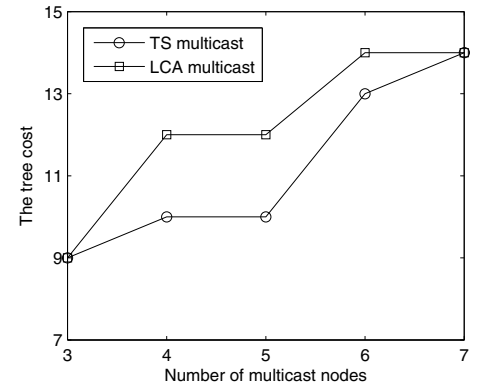

(a)

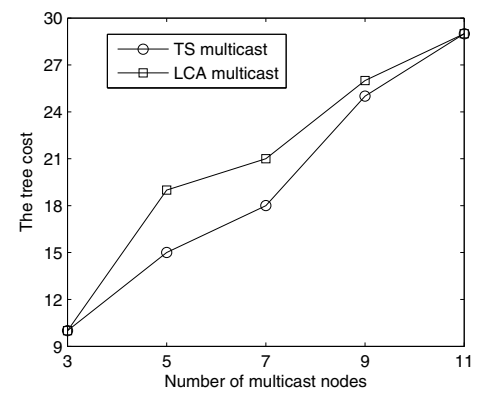

(b)

Fig. 2. Comparison of TS multicast and LCA multicast in terms of the total channel conflict in: (a) a WMN of 11 nodes; (b) a WMN of 23 nodes.

continuous iterations without improving the present optimal solution is reached. Without loss of generality, we assume each mesh router has two radio network interface cards: one for transmission and the other for reception. We assume there are 3 orthogonal channels as the case in 802.11 wireless network. We evaluate both algorithms on two different network topologies: one consists of 11 nodes and 20 links and the other consists of 23 nodes and 34 links. The metrics that we have evaluated include the total channel conflict and the tree cost.

We have compared the TS multicast algorithm with the LCA multicast algorithm on various size of multicast groups. In the WMN of 11 nodes, the size ranges from 3 to 7 while in the WMN of 23 nodes it ranges from 3 to 11 . Fig. 1 and Fig. 2 show the comparison results in terms of the total channel conflict and the tree cost, respectively. From Fig. 1, we can see that in both networks, our TS multicast algorithm can find the multicast trees with less channel conflict than the trees obtained by the LCA multicast algorithm. In the network of 11 nodes, the TS multicast algorithm can always find the conflictfree multicast trees while LCA cannot. Furthermore, with the increase in the multicast group size, more and more conflict occurs on the LCA multicast trees. Fig. 2 shows that the cost of our TS multicast trees is lower than the cost of the LCA multicast trees when the multicast group size is beyond 3 . It means that the TS multicast trees consume less radio network resouces. To sum up, our TS multicast algorithm can find the multicast trees which log less channel conflict and lower cost while satisfying the delay constraint.

\section{CONCLUSION}

The wireless mesh networks have seen various collaborative multimedia applications which require efficient information delivery service from a designated source to multiple receivers. A multicast tree with orthogonal channels appropriately assigned is preferred to support this service. However, the optimal multicast routing and channel assignment problem is proved to be NP-hard. Unfortunately, so far little work has been done on it. This paper presents a tabu search based joint multicast routing and channel assignment algorithm for wireless mesh networks. It aims to discover a delay-bounded minimum-interference low cost multicast tree. We believe that the synergy achieved by combining the strong search capability of TS and the effective channel assignment results in the improved quality of solution. We compare the performance of the proposed algorithm with the prestigious LCA multicast algorithm. Experimental results demonstrated that our TS multicast algorithm is capable of finding the multicast trees which have both less channel conflict and lower cost (i.e., consuming less radio network interfaces) than the multicast trees produced by the LCA multicast algorithm.

\section{ACKNOWLEDGMENT}

This work was supported by the Engineering and Physical Sciences Research Council (EPSRC) of UK under Grant EP/E060722/1.

\section{REFERENCES}

[1] Akyildiz, I., Wang, X., Wang, W.: Wireless Mesh Networks: a Survy. Comput. Netw. 47, 445-487 (2005)

[2] Subramanian, A., Gupta, H., Das, S.: Minimum Interference Channel Assignment in Multi-Radio Wireless Mesh Networks. In: 4th Annual IEEE Communications Society Conference on Sensor, Mesh, and Ad Hoc Communications and Networks (SECON), pp. 481-490 (2007)

[3] Wang, X., Cao, J., Cheng, H., Huang, M.: QoS Multicast Routing for Multimedia Group Communications Using Intelligent Computational Methods. Comput. Comm. 29, 2217-2229 (2006)

[4] Parsa, M., Zhu, Q., Garcia-Luna-Aceves, J.: An Iterative Algorithm for Delay-constrained Minimum-cost Multicasting. IEEE/ACM Trans. Netw. 6, 461-474 (1998)

[5] Jain, K., Padhye, J., Padmanabhan, V., Qiu, L.: Impact of Interference on Multi-hop Wireless Network Performance. In: 9th ACM Annual International Conference on Mobile Computing and Networking (MobiCom), pp. 66-80 (2003)

[6] Zeng, G., Wang, B., Ding, Y., Xiao, L., Mutka, M.: Multicast Algorithms for Multi-Channel Wireless Mesh Networks. In: 15th IEEE International Conference on Network Protocols (ICNP), pp. 1-10 (2007)

[7] Glover, F.: Tabu search: Part I, ORSA J. Comput. 1, 190-206 (1989)

[8] Cordeiro, C., Gossain, H., Agrawal, D.: Multicast over Wireless Mobile Ad Hoc Networks: Present and Future Directions. IEEE Netw. 17, 52-59 (2003)

[9] Habib, Y., Abdulaziz, A., Sadiq, M., Muhammad, A.: QoS-driven Multicast Tree Generation using Tabu Search. Comput. Commun. 25, 1140-1149 (2002)

[10] Din, D.: Anycast Routing and Wavelength Assignment Problem on WDM Network. IEICE Trans. Commun. E88-B, 3941-3951 (2005)

[11] Ahn, C., Ramakrishna, R.: A Genetic Algorithm for Shortest Path Routing Problem and the Sizing of Populations. IEEE Trans. Evol. Comput. 6, 566-579 (2002)

[12] Thai, M., Li, Y., Du, D.: A Combination of Wireless Multicast Advantage and Hitch-hiking. IEEE Commun. Lett. 9, 1037-1039 (2005) 